\title{
Struma ovarii accompanied by mature cystic teratoma of the other ovary: A case report and literature review
}

\author{
PENG-FEI QIAO, YANG GAO and GUANG-MING NIU \\ Department of Magnetic Resonance Imaging, Affiliated Hospital of Inner Mongolia Medical University, \\ Hohhot, Inner Mongolian Autonomous Region 010050, P.R. China
}

Received May 16, 2014; Accepted January 29, 2015

DOI: $10.3892 / \mathrm{ol} .2015 .3029$

\begin{abstract}
Struma ovarii, as a monodermal variant of ovarian teratoma, constitutes $<3 \%$ of ovarian teratomas. It is difficult determine a diagnosis prior to surgery. The current study reports an unusual case of struma ovarii occurring in a 49 year-old female, which was accompanied by mature cystic teratoma involving the other ovary. The final pathological diagnosis was confirmed as struma ovarii based on the typical morphology of the thyroid follicles and the results of immunohistochemical staining. The bilateral tumors were resected and follow-up examinations were planned at four month intervals. At the time of writing, the patient was well and no tumor recurrence had been identified.
\end{abstract}

\section{Introduction}

Struma ovarii (SO) originates from ovarian primitive germ cells, and is classified as a single layer, highly specific mature ovarian teratoma (1). In cases where the thyroid component of the tumor accounts for $>50 \%$ of all tumor tissue, or $<50 \%$ but with symptoms of hyperthyroidism, this may be diagnosed as SO (2). SO is a rare condition, accounting for $0.3 \%$ of ovarian tumors and $2.7 \%$ of ovarian teratomas; $5-10 \%$ of tumors become malignant (3). It predominantly occurs between 30-50 years of age, most often in the unilateral ovary, and occasionally in combination with contralateral ovary mature teratoma and cystadenoma (4). Frequently, a lack of clinical characteristics are present; certain patients experience peritoneal effusion and/or, pleural effusion [false Meigs sign (5)], and elevated serum CA125 is observed in a number of cases. However, the occurrence of pleural effusion is not necessarily indicative of malignant manifestation (5).

Correspondence to: Professor Guang-Ming Niu, Department of Magnetic Resonance Imaging, Affiliated Hospital of Inner Mongolia Medical University, 1 Tong Dao Street, Hohhot, Inner Mongolian Autonomous Region 010050, P.R. China

E-mail: niugming@gmail.com

Key words: magnetic resonance imaging manifestations, differential diagnosis, struma ovarii
In $5 \%$ patients with thyroid tissue differentiation, mature thyroid hyperfunction may be observed (6). Written informed consent was obtained from the patient.

\section{Case report}

A 49 year-old female patient was admitted to The Affiliated Hospital of Inner Mongolia Medical University (Hohhot, China) with an adnexa uteri tumor, which was identified by magnetic resonance imaging (MRI). Approximately two years previously, the patient incidentally identified a lump, which was $30 \mathrm{~mm}$ in diameter; no pain was experienced and the mass was palpable. After a further year, an additional lump was identified in the right lower abdomen. The bilateral masses increased gradually from this time, and this was accompanied by intermittent abdominal pain over the course of the disease. Gynecological examination revealed a normal uterine size (diameter, $\sim 50 \mathrm{~mm}$ ), a cystic and solid neoplasm of $\sim 8 \mathrm{~cm}$ in diameter in the right adnexa uteri, and a cystic and solid neoplasm of $\sim 7 \mathrm{~cm}$ in the left adnexa uteri. The two masses were movable. The family history revealed no previous instances of this condition. Physical examination revealed no abnormalities.

MRI showed abnormal signals in the bilateral adnexa uteri (Figs. 1 and 2). During surgery to resect the bilateral masses, the uterus was observed to be of normal size with a diameter of $53 \mathrm{~mm}$, the tumor on the right ovary was $\sim 73 \times 58 \mathrm{~mm}$ in diameter and was a complete capsule with a smooth surface, containing a colorless liquid. The left ovarian mass was $\sim 75 \times 87 \mathrm{~mm}$ in diameter and was a complete capsule, with a surface scattered with small blister-like nodules. Prior to surgery, the mass in the right ovary was misdiagnosed as serous cystadenoma. However, following resection of the two masses histological and immunological analysis were performed, which revealed the pathological diagnosis of struma ovarii in the right ovary and mature cystic teratoma in the left ovary (Fig. 3). The tumor in the right ovary consisted of thyroid tissue and stroma, with abundant blood vessels and fibrous tissue. The tumor in the left ovary was a cystic solid lesion, with thick regular borders and fatty components were identified within the cyst. Follow-up examinations every four months were planned. At the time of writing, the patient was well and no recurrence had been identified. 
A

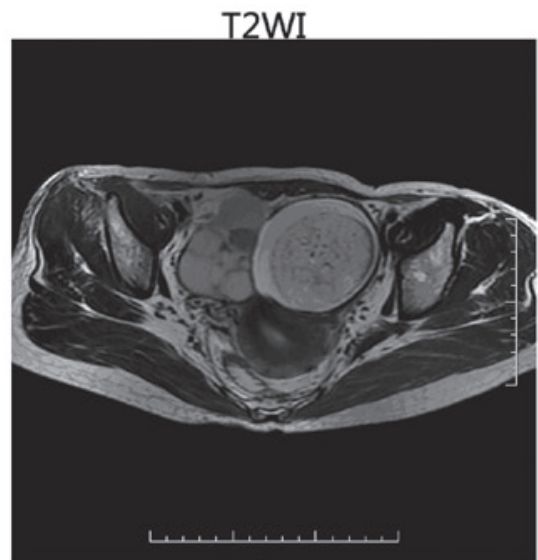

B

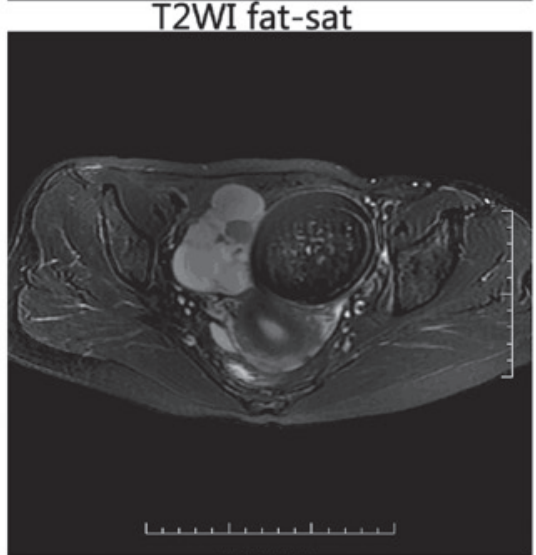

C

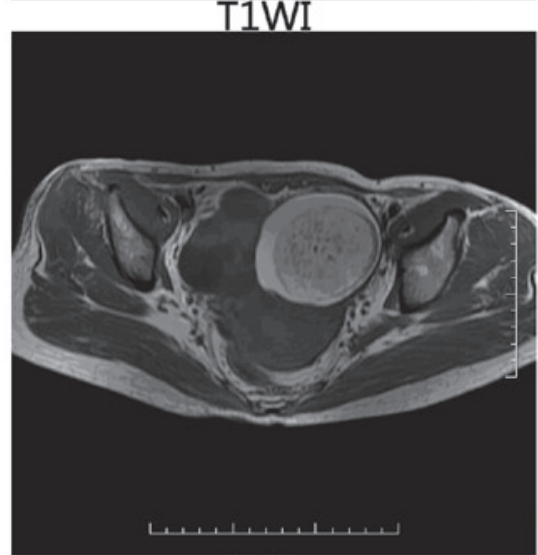

DWI

D

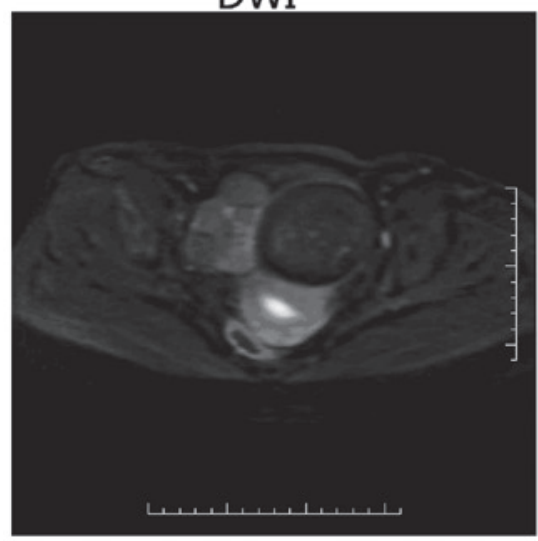

Figure 1. Plain magnetic resonance imaging. (A) Mixed T2 signals were observed in the right ovary, with a clear boundary, inside separations, as well as long T2 signals in the left ovary. (B) Low signals were observed in the fat suppressed sequences. (C) Mixed T1 signals were observed in the right ovary, with a clear boundary and inside separations, as well as short T1 signals in the left ovary. (D) When compared with other ovarian tumors, no specific signs were identified on DWI. DWI, diffusion weighted imaging.

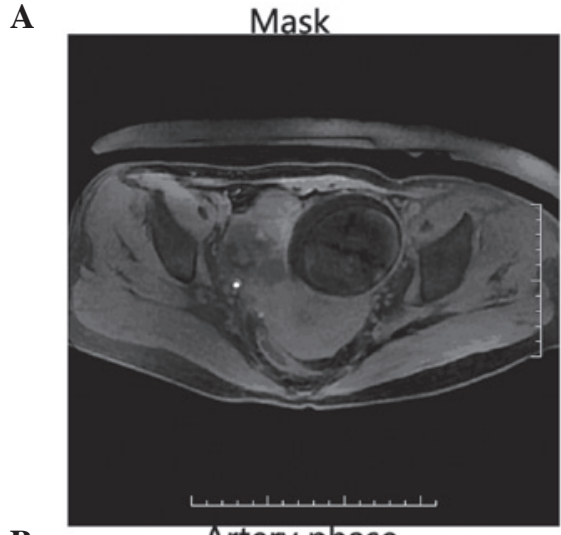

B

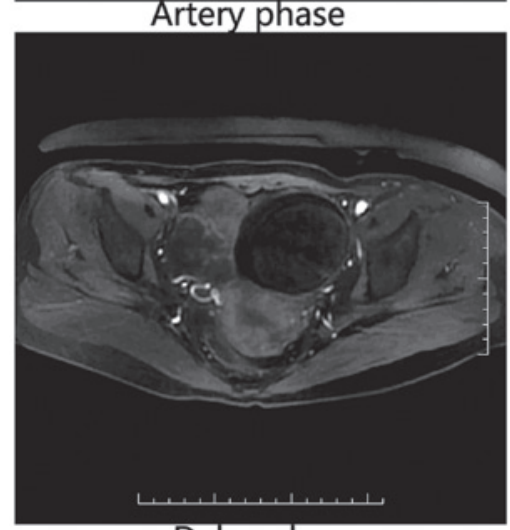

C

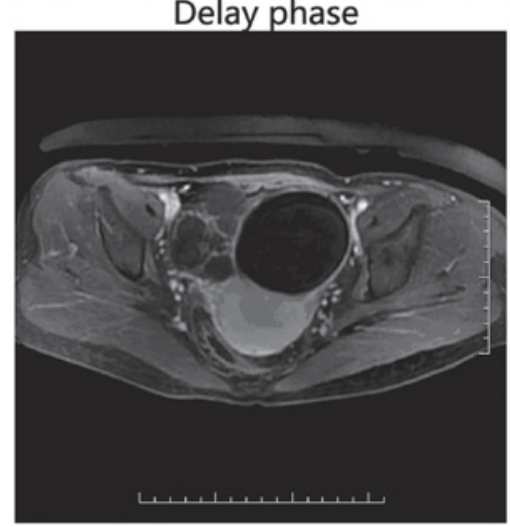

Figure 2. Enhanced magnetic resonance imaging. (A) The ovaries without the injection of contrast agent in the mask phase. (B) The right ovary mass with inhomogeneous enhancement, and the left ovary mass, which exhibited no enhancement in the artery phase. (C) The enhancement of the right ovary mass decreased in the delay phase.

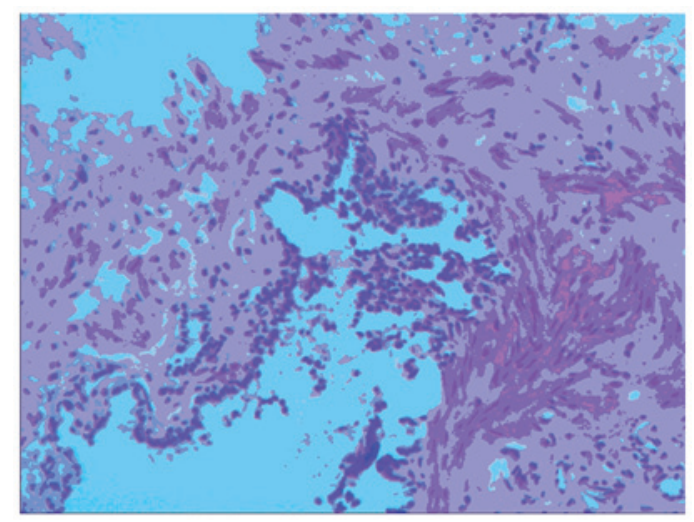

Figure 3. Tumor was composed of thyroid follicle tissues, which contained gelatinous thyroid colloids, and follicular epithelium, which consisted of cuboidal epithelium or columnar epithelium without cell heteromorphism. 


\section{Discussion}

$\mathrm{SO}$ is a rare ovarian benign tumor, classified as single layer ovarian teratoma by the WHO classification (7). SO is easily misdiagnosed prior to surgery as the final diagnosis is dependent on pathological analysis. Pathologically, all or the majority of the tumor tissue was a particular type of mature teratoma, which was composed of thyroid tissue (8). MRI may be used to show the complex cystic and solid mass in cases of SO, with variable signals, and the observable characteristics may be as follows: i) A cystic and solid mass with clear boundaries, composed of cystic intervals or where cystic components predominate indicates the most common type of $\mathrm{SO}$; ii) multiple cysts show different signals from high to low on MRI, but are homogeneous. Tumor hemorrhage may be observed on short T1 and short T2, or short T1 and long T2 signals. On T2 weighted imaging (WI), no chemical shift artifact is present. Okada et al (9) demonstrated that the low signal observed for the mass on T2WI, occasionally referred to as the vacuum phenomenon, is associated with the viscous fluid within cysts, which is a specific indicator of SO; iii) the wall and interval of cyst may be thick $(\sim 5 \mathrm{~mm})$; nodules are rare, and where present, are significantly enhanced in the contrast MRI scan; and iv) in instances where the lump is solid, it may also be enhanced.

The MRI revealed mixed T1 and T2 signals; the SO had short T2 signals, uneven thickness, and was enhanced in the contrast MRI. A region of the cyst exhibited short T2 and marginally long T1 signals, and the signal was similar in the images acquired using fat-suppressed and non-fat-suppressed sequences, and was not enhanced on the contrast MRI scan. This was proposed to be the vacuum phenomenon, as discussed above. The contralateral ovarian lesion was a typical cystic teratoma, which exhibited high T1, T2 based mixed signals; the high signals were suppressed and lower than the signals of the tissues on fat-suppressed sequence, and were not enhanced throughout the dynamic enhanced MRI scan.

In the differential diagnosis, SO must be differentiated from other cysts and cystic masses of the adnexa uteri including ovarian serous cystadenoma, mucinous cystadenoma and cystadenocarcinoma. Ovarian serous cystadenomas are predominantly single cysts with abnormal long T1 and long T2 signals inside. They differ from SO in that the wall and interval of the cyst is thicker and only certain SOs exhibit extremely low signals on T2WI. The present case was initially misdiagnosed as serous cystadenoma prior to surgery. Mucinous cystadenoma is predominantly multicystic, with a variable signal; high signals are usually observed on T2WI. The cyst wall and interval are consistently thinner than that of SO. No obvious enhancement is observed on the contrast MRI scan. Cystadenocarcinoma contains more solid constituents than SO, the cystic wall is uneven and is accompanied by nodules in the wall. Therefore, MRI may aid in distinguishing SO from other carcinomas.

In conclusion, a diagnosis of SO must be considered in females of child-bearing age, where a single solid and cystic mass is observed in adnexa uteri, with a lobulated appearance, clear boundaries and a thick cystic wall. Certain cases of SO exhibit calcification, with a cystic region showing long T1 and long T2 signals on plain MRI; additionally, a significantly enhanced solid component, cystic wall and septum may be observed on contrast MRI. Therefore, the MRI features of SO may aid with pathological diagnosis prior to surgery, in patients with ovarian tumors that exhibit atypical features.

\section{References}

1. Raina A, Stasi G, Monzio Compagnoni B, et al: Struma ovarii - a rare gynecological tumor. Acta Oncol 36: 533-534, 1997.

2. Krishnamurthy A, Ramshankar V, Vaidyalingam V and Majhi U: Synchronous papillary carcinoma thyroid with malignant struma ovarii: A management dilemma. Indian J Nucl Med 28: 243-245, 2013.

3. Outwater EK, Siegelman ES and Hunt JL: Ovarian teratomas: tumor types and imaging characteristics. Radiographics 21: 475-490, 2001.

4. Nurliza Binti Md Nor, Kusumoto T, Inoue S, et al: Three cases of struma ovarii underwent laparoscopic surgery with definite prepperative diagnosis. Acta Med Okayama 67: 191-195, 2013.

5. Mostaghel N, Enzevaei A, Zare K and Fallahian M: Struma ovarii associated with Pseudo-Meig's syndrome and high serum level of CA125; a case report. J Ovarian Res 21: 10, 2012.

6. Teale E, Gouldesbrough DR and Peacey SR: Graves disease and coexisting struma ovarii:struma expression of thyrotropin receptors stimulating antibodies. Thyroid 16: 791-793, 2006.

7. D'Antonio A, Caleo A, Caleo O, et al: Hashimoto thyroiditis as a manifestation of struma ovarii. Endocrinologist 20: 220-221, 2010.

8. Matsuda K, Maehama T and Kanazawa K: Malignant struma ovarii with thyrotoxicosis. Gynecol Oncol 82: 575-577, 2001.

9. Okada S, Ohaki Y, Kawamura T, et al: Cystic struma ovarii: imaging findings. J Comput Assist Tomogr 24: 413-415, 2000. 